\title{
Polifluoruro de amonio como alternativa de acondicionamiento para laminados ultradelgados de disilicato de litio. Reporte de caso.
}

\section{Ammonium polyfluoride as an alternative for the surface conditioning of ultrathin lithium disilicate veneers. A case report.}

\author{
Fernando Grandon ${ }^{1 *}$, Nicole Santana ${ }^{1}$, Natalia Marcus ${ }^{1}$, María Muster ${ }^{2}$, Blas Galdames ${ }^{1}$, Michael Wendler ${ }^{1}$
}

1. Departamento de Odontología Restauradora, Facultad de Odontología, Universidad de Concepción, Chile.

2. Facultad de Odontología, Universidad Andrés Bello, Chile.

* Correspondencia Autor: Dr. Michael Wendler | Departamento de Odontología Restauradora, Facultad de Odontología, Universidad de Concepción | Dirección: Roosevelt 1550, Concepción, Chile |E-mail: mwendler@udec.cl| Teléfono: +56412661786 - +56412204481

Trabajo recibido el 26/01/2020.

Trabajo revisado 08/04/2020

Aprobado para su publicación el 07/07/2020

\begin{abstract}
RESUMEN
Los laminados vitrocerámicos ultradelgados constituyen una alternativa conservadora para la resolución de alteraciones estéticas. Sin embargo, su acondicionamiento con ácido fluorhídrico suele no estar exento de complicaciones, por lo que el uso de un sistema autograbante en base a polifluoruro de amonio (Monobond Etch\&Prime, MEP) permite disminuir el riesgo de sobregrabado, simplificando la técnica. El presente reporte presenta el seguimiento a dieciocho meses de un caso clínico resuelto mediante laminados vitrocerámicos acondicionados únicamente con MEP. Caso. Paciente joven con alteraciones estéticas en el sector anterosuperior. Se realizaron preparaciones conservadoras para la confección de laminados ultradelgados en disilicato de litio (e.maxPress). Las vitrocerámicas fueron acondicionadas sólo con MEP y se cementaron con Variolink Esthetic LC. Al año y medio las restauraciones se observaron indemnes, manteniendo un buen ajuste marginal y ausencia de tinciones. Conclusiones. MEP aparece como una alternativa prometedora para reemplazar al ácido fluorhídrico en la cementación de laminados vitrocerámicos.

PALABRAS CLAVE:
\end{abstract}

Laminado ultradelgado; Vitrocerámica; Polifluoruro de amonio; Acondicionador cerámico autograbante.

Int. J. Inter. Dent Vol. 14(1); 44-47, 2021.

\section{ABSTRACT}

Ultrathin glass-ceramic veneers are a conservative approach for the restorative treatment of aesthetic disorders in the anterior region. However, surface conditioning with hydrofluoric acid of the thin structures can be usually a challenging step. Therefore, the use of a self-etching ceramic primer containing ammonium polyfluoride (Monobond Etch\&Prime, MEP) offers a simplified technique with a reduced risk of overetching. Here we present an 18-month follow-up of ultrathin glass-ceramic veneers conditioned with MEP only. Case. Young female patient with an aesthetic disorder in the front region. The four upper incisors were conservatively prepared for ultrathin lithium disilicate (e.maxPress) veneers. Pre-treatment of the glass-ceramics consisted only of MEP application, followed by adhesive cementation with Variolink Esthetic LC. At the 1.5year recall, the restorations appeared undamaged, with no marginal gap or staining. Conclusions. MEP constitutes a promising alternative to hydrofluoric acid for the adhesive cementation of glass-ceramic veneers.

KEY WORDS:

Ultrathin veneer; Glass-ceramic; Ammonium polyfluoride; Self-etching ceramic primer.

Int. J. Inter. Dent Vol. 14(1); 44-47, 2021.

\section{INTRODUCCIÓN}

Los laminados cerámicos representan una valiosa herramienta para dar solución a pacientes afectados por problemas funcionales o estéticos bajo un enfoque indirecto de mínima intervención ${ }^{(1)}$. Las excelentes propiedades mecánicas de las vitrocerámicas contemporáneas, así como el mejoramiento continuo de los sistemas de fijación, han permitido extender el rango de aplicación de los laminados, posicionándolos como una alternativa más conservadora y eficiente a la prótesis fija convencional ${ }^{(2)}$. Es más, la unión adhesiva de las vitrocerámicas al sustrato dentario ha demostrado incrementar su resistencia flexural incluso a valores similares a los de cerámicas de alto rendimiento como la zirconia(3). Es por ello, que tanto el acondicionamiento previo de las superficies, como los materiales y la técnica de cementación, resultan fundamentales para el éxito clínico de este tipo de tratamientos ${ }^{(4)}$. El método de acondicionamiento más efectivo para lograr una buena unión adhesiva a los sustratos con alto contenido vítreo sigue siendo el grabado con ácido fluorhídrico (HF) y la aplicación de un agente de unión silano ${ }^{(4)}$. Sin embargo, su uso no está exento de controversias por sus potenciales efectos negativos sobre la salud, dada su alta toxicidad y volatilidad(5), así como por el alto riesgo que supone su uso en la reparación intraoral de cerámicas. Por lo tanto, el desarrollo de protocolos libres de HF ha sido 
uno de los ejes principales de investigación en esta área.

Entre las diferentes alternativas que se han propuesto para reemplazar el acondicionamiento con HF durante los últimos años, destaca el uso de agentes autograbantes en base a fluoruros ${ }^{(6,7)}$ capaces de lograr en un mismo tiempo clínico patrones microretentivos y unión química al sustrato. Uno de los exponentes más conocidos de esta nueva generación de primers cerámicos es el Monobond Etch and Prime (MEP, Ivoclar Vivadent, Schaan, Lichtenstein). En él se combinan el efecto grabador del polifluoruro de amonio con la capacidad de unión química del agente de unión silano al componente vítreo de la cerámica ${ }^{(8)}$, en una técnica de aplicación simplificada. Pese a que los patrones de grabado logrados por el polifluoruro de amonio son mucho menos pronunciados que los generados por el $\mathrm{HF}^{(9)}$, diversos estudios in vitro han demostrado que los valores de resistencia adhesiva son equivalentes a los obtenidos con la técnica convencional( ${ }^{(7,10)}$, mostrando incluso una mejor estabilidad adhesiva tras aplicación de termociclaje ${ }^{(11,12)}$.

El objetivo del presente reporte es presentar un caso clínico de alta complejidad estética resuelto mediante laminados ultradelgados en base a disilicato de litio acondicionados únicamente con MEP. Este tipo de restauraciones supone un gran desafío, puesto que su sobrevida en el tiempo depende en gran parte de la unión adhesiva al sustrato dentario. El exitoso seguimiento a dieciocho meses aporta evidencia sobre la efectividad clínica de este nuevo tipo de acondicionamiento.

\section{REPORTE DEL CASO}

Paciente de género femenino, 26 años de edad, sin antecedentes sistémicos relevantes, consultó por disconformidad con el color de sus dientes, morfología de sus incisivos superiores y diastemas en el sector ánterosuperior. El examen clínico arrojó elementos segregativos en la línea de la sonrisa, dientes con saturación acentuada, marcados diastemas e incisivos laterales con leve forma conoide (Figura 1). Por otra parte, presentaba apiñamiento anteroinferior, por lo cual recibe la recomendación de terapia ortodóncica previo al tratamiento restaurador, aunque la paciente relató en ese momento no poder realizarla en el corto plazo. Por lo tanto, se planificó como tratamiento laminados vitrocerámicos ultradelgados, con el objetivo de mejorar su sonrisa preservando al máximo su estructura dentaria. Además del registro fotográfico, se confeccionó encerado diagnóstico en el que se redistribuyó el espacio de los cuatro incisivos maxilares para realizar posteriormente un ensayo restaurador (mock-up) con resina bisacrílica (Protemp 4, 3M, Seefeld, Alemania) para la aprobación final de la paciente (Figura 2).

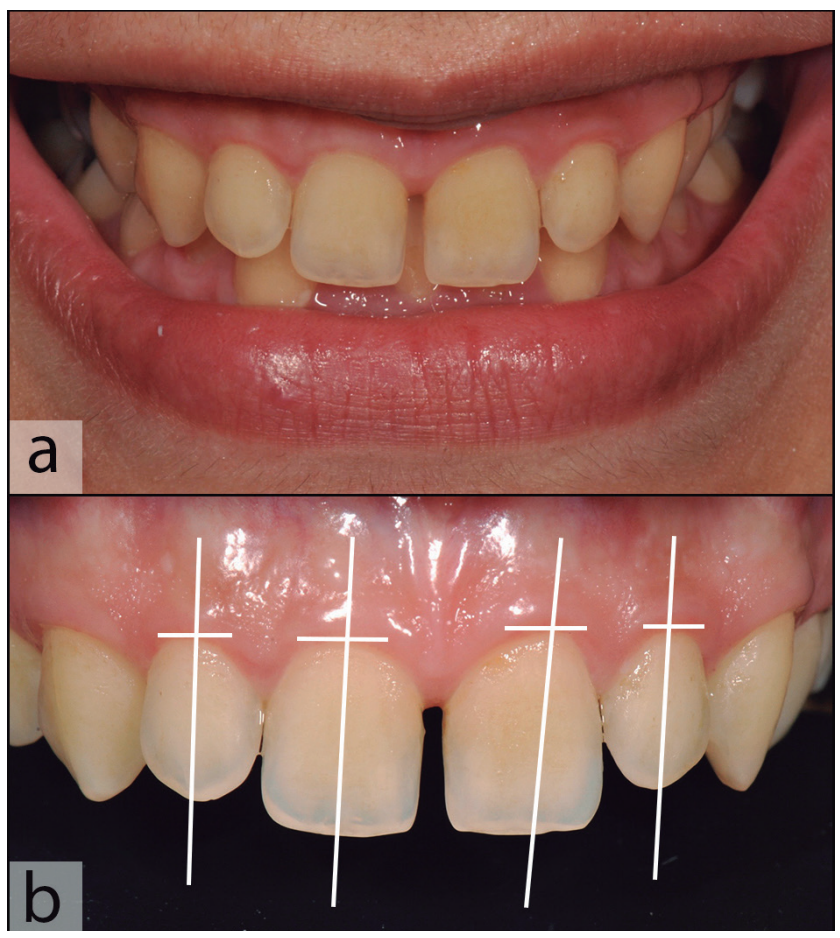

Figura 1. Situación inicial. En a se observan los elementos segregativos que alteran la línea de la sonrisa, con presencia de un diastema importante entre ambos centrales y laterales levemente conoides. A esto se suma la alta saturación de dientes maxilares y mandibulares. En b se observa además una alteración en los ejes axiales de los anterosuperiores, con 1.2 y 1.1 distalizados.

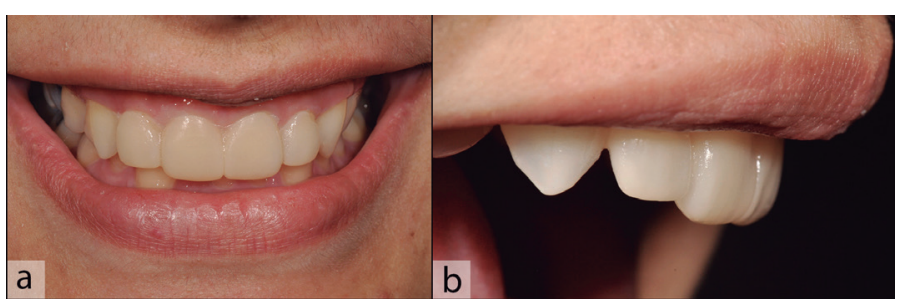

Figura 2. Transferencia del encerado diagnóstico (mock-up). En a se presenta la vista frontal, donde se observa3 la redistribución de espacios. Se aumentó levemente el ancho mesiodistal de los laterales, lo que fue compensado por un cambio en el eje axial de los incisivos centrales. En b se muestra la vista lateral. Luego del primer prototipo se optó por aumentar el largo de los incisivos laterales, de modo de compensar el gran volumen que presentan los caninos.

Previo al tratamiento rehabilitador se realizó un blanqueamiento selectivo en casa con peróxido de carbamida al 15\% (Opalescence PF, Ultradent, South Jordan, Estados Unidos), 2 horas diarias por dos semanas en dientes anteroinferiores, caninos y premolares maxilares. Una vez obtenida la mejora de color en dichos dientes, se utilizó el encerado diagnóstico como guía de desgaste para los anterosuperiores, obteniendo así preparaciones con los espesores mínimos necesarios (Figura 3). La impresión fue tomada con silicona por adición (President, Coltene, Altstätten, Suiza) solicitando al laboratorio la confección de laminados en disilicato de litio IPS e.max Press (Ivoclar Vivadent) de alta translucidez (HT) mediante la técnica de inyección. Se realizó una prueba clínica en seco para corroborar ajuste y forma y luego se simuló el efecto del cemento sobre el resultado estético final mediante Variolink Esthetic LC Try-In color Light (Ivoclar Vivadent) (Figura 3). Tras eliminar completamente la pasta de prueba con spray aire-agua, los laminados fueron acondicionados con MEP (Figura 4) siguiendo las indicaciones del fabricante: aplicación activa con un micropincel por $20 \mathrm{~s}$, seguidos por $40 \mathrm{~s}$ adicionales para permitir la reacción con la superficie (Figura 5). Las superficies cerámicas fueron luego lavadas con pulverización aireagua para eliminar el MEP y luego sólo aire para su secado. Por otra parte, las superficies dentarias se acondicionaron con ácido ortofosfórico (Ultraetch, Ultradent) y fueron imprimadas con el sistema adhesivo Tetric $\mathrm{N}$-Bond Universal (Ivoclar Vivadent), el cual se mantuvo sin polimerizar hasta el momento de la fijación. La cementación se realizó con Variolink Esthetic LC (Ivoclar Vivadent) color Light. El cemento fue polimerizado inicialmente con lámpara de alta potencia por $2 \mathrm{~s}$ y los restos removidos con hoja de bisturí $\mathrm{N}^{0} 12$. Se aplicó un segundo ciclo de fotopolimerización de $60 \mathrm{~s}$ por cada diente tras aplicación previa de glicerina Liquid Strip (Ivoclar Vivadent) sobre los márgenes, para asegurar la eliminación de la capa inhibida de oxígeno.

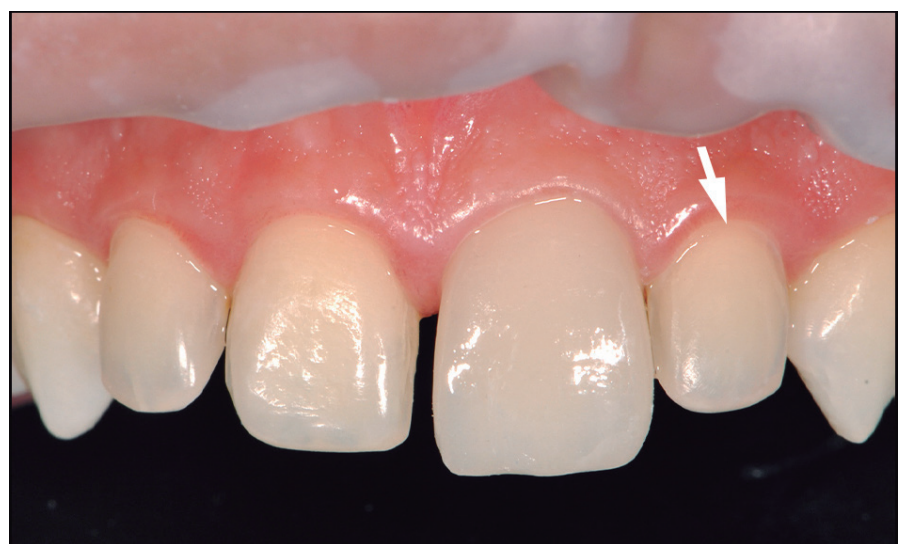

Figura 3. Preparaciones dentarias y prueba en boca del laminado de 2.1 Estas se realizaron utilizando como guía matrices de silicona obtenidas a partir del encerado diagnóstico. El desgaste se hizo sólo a expensas del esmalte, no superando los $0.3 \mathrm{~mm}$, dejando una terminación yuxtagingival (como se indica mediante la flecha sobre 2.2). Como se observa en 2.1, la prueba en boca de los laminados vitrocerámicos se realizó utilizando una pasta de prueba (try in) que permitió evaluar el efecto del cemento sobre el color y la translucidez final de la restauración. 


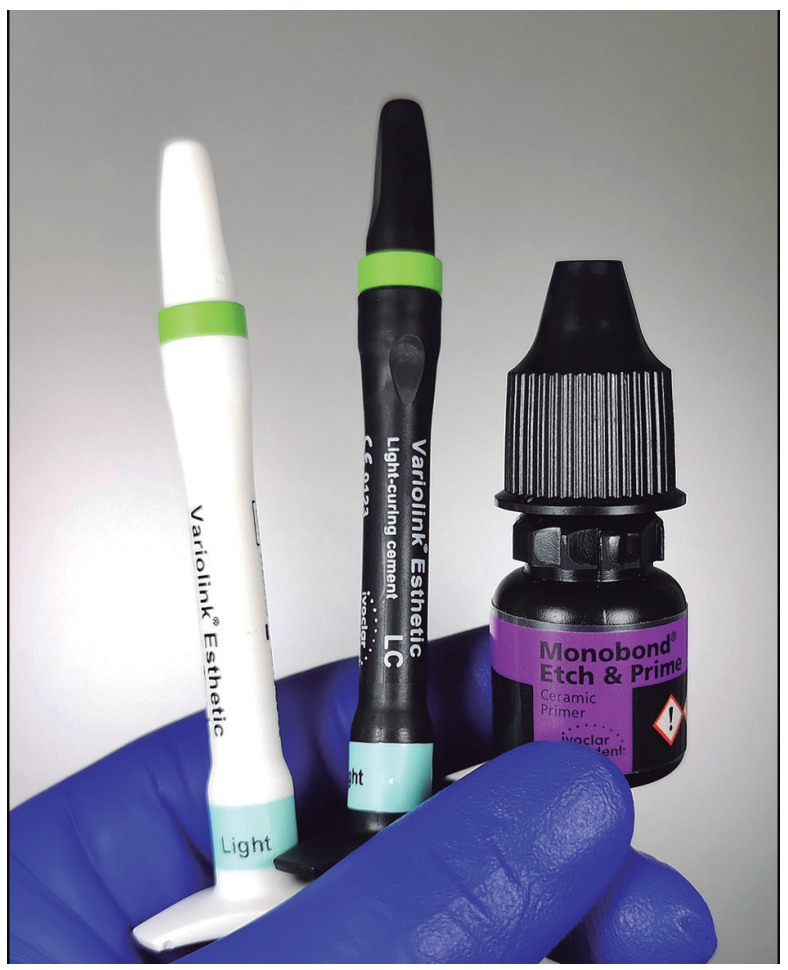

Figura 4. Materiales utilizados para el acondicionamiento y cementación adhesiva de los laminados. El MEP contiene en una botella el primer autograbante polifluoruro de amonio y el agente de unión silano. El sistema de cementación fotopolimerizable Variolink Esthetic consiste en la pasta de prueba o try in (jeringa blanca) y el agente cementante (jeringa negra).

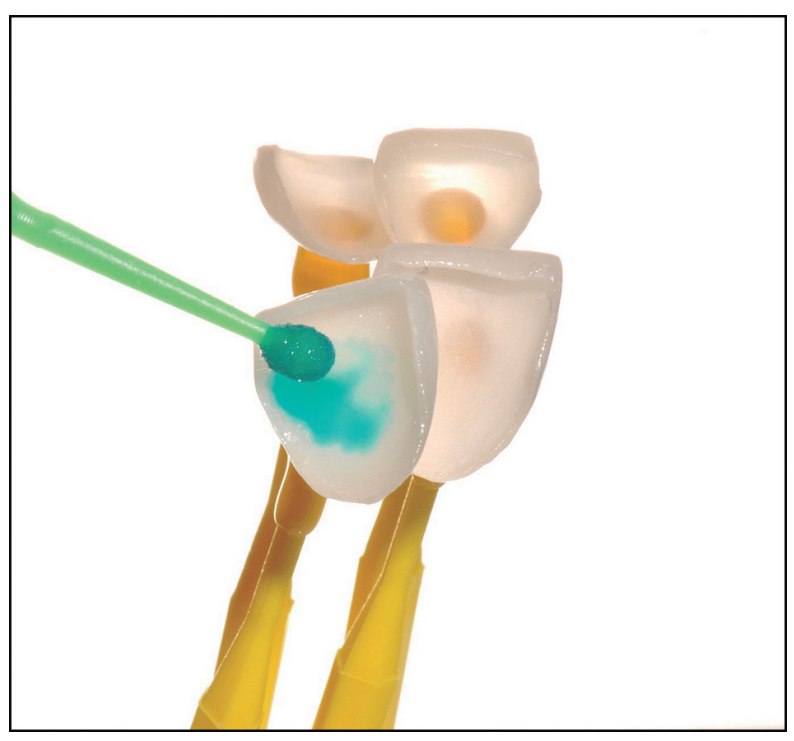

Figura 5. Acondicionamiento de los sustratos vitrocerámicos con MEP. Los laminados fueron montados sobre sujetadores de precisión para facilitar su manipulación. Se realizó una aplicación activa con micropincel por $20 \mathrm{~s}$, cuidando de no invadir los márgenes ni las zonas vestibulares de los laminados. Luego se dejó actuar por $40 \mathrm{~s}$ para asegurar el correcto acondicionamiento de la superficie vítrea y la condensación de la capa de silano sobre ella, tras lo cual se procedió a lavar profusamente y secar con aire.

El resultado final se muestra en la Figura 6. Pese a la mínima intervención realizada, se consiguió armonizar la sonrisa, devolviendo los ejes correctos y cerrando adecuadamente los diastemas. La combinación de una cerámica de alta translucencia en espesores mínimos y el correcto manejo del color del cemento permitieron un adecuado aspecto final, manteniendo la naturalidad. Por otra parte, la terminación yuxtagingival (Figura 3) y el adecuado ajuste y control de márgenes durante la cementación favorecieron la mantención de la salud periodontal, como se puede observar en las fotografías del control a los 18 meses (Figura 7). Para sorpresa de los autores, la paciente acudió a este último control con aparatología de ortodoncia instalada sobre las carillas. Si bien en un inicio ella había rechazado esta propuesta, el resultado obtenido en el sector anterosuperior mejoró su disposición frente a la odontología, por lo que tomó la decisión de mejorar el apiñamiento antes descrito. Los brackets fueron instalados al año de cementadas las carillas, por lo que ya llevaban 6 meses en boca cuando acudió al control. Pese a la fuerte tracción que los alambres ejercen a través de los brackets sobre los laminados cerámicos, estos se encontraron indemnes, lo que resalta aún más la calidad de la unión lograda al sustrato dentario.

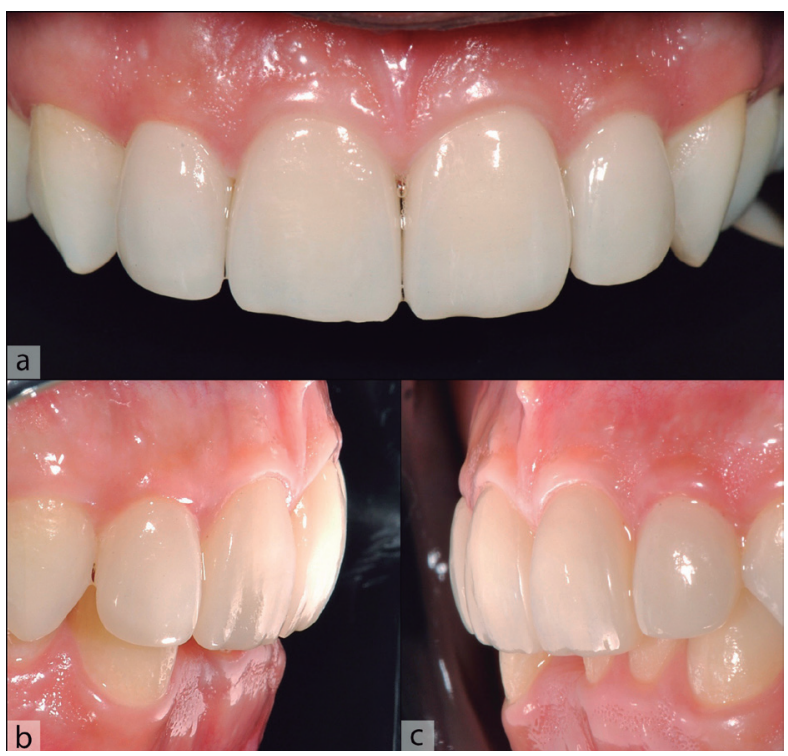

Figura 6. Resultado final, control a la semana. Los laminados vitrocerámicos logran devolver adecuadamente la armonía al sector anterior, recuperando las proporciones dentarias y cerrando los diastemas. Se observa además el equilibrio biológico logrado con los tejidos de soporte gracias al excelente ajuste de los laminados y la ubicación yuxtagingival de su margen cervical.

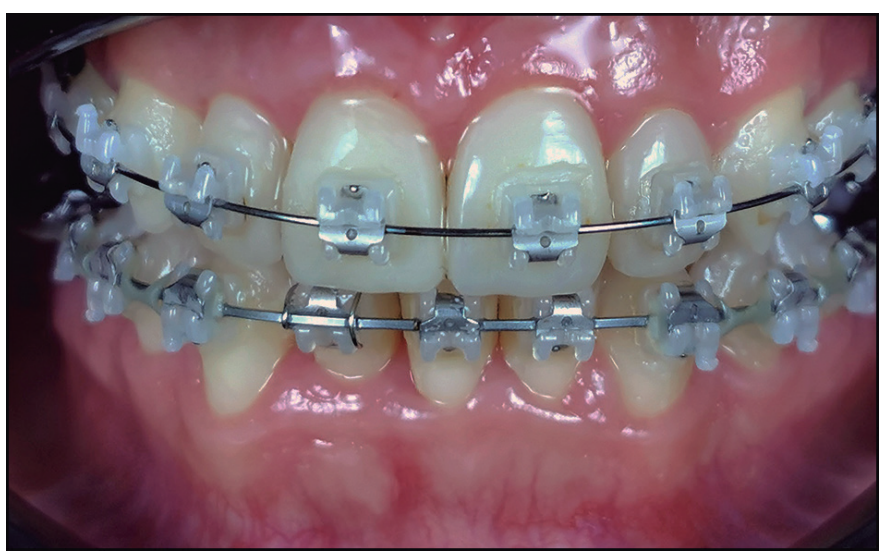

Figura 7. Control a los 18 meses. Pese a la instalación de aparatos de ortodoncia sobre la superficie de los laminados vitrocerámicos, estos se mantienen funcionando adecuadamente. No se observan tinciones de los márgenes cervicales y la salud periodontal está conservada.

\section{DISCUSIÓN}

La indicación de carillas ultradelgadas, también denominadas "lentes de contacto dentales", permite a través de un abordaje de mínima intervención la restitución no sólo de la forma y contornos dentarios, sino también la redistribución de espacios, devolviendo la simetría y armonía a la sonrisa. Como se puede observar en el presente caso, los desgastes necesarios alcanzan sólo las décimas de milímetros, conservando la mayor parte de la estructura adamantina y preservando de esta manera íntegros los componentes ópticos del diente. Esto implica, a su vez, un gran desafío en términos de manejo de la translucidez y el color de la restauración, tanto por parte del técnico como durante el proceso de 
cementación adhesiva. La adecuada manipulación de los laminados en esta última etapa es otro factor crítico, especialmente cuando se deben aplicar y lavar sucesivamente agentes acondicionadores e imprimadores. En este contexto, el uso de MEP en reemplazo del HF disminuye el riesgo sobregrabar y de esta manera debilitar una estructura de por sí muy delgada. Por otra parte, la aplicación del ácido y el agente de unión silano en un solo tiempo reduce la sensibilidad de la técnica y la posibilidad de contaminación por humedad entre ambos pasos ${ }^{(10)}$. Se logra así estandarizar de buena manera el acondicionamiento del sustrato y mejorar su rendimiento clínico en el tiempo.

Si bien los patrones de grabado ácido logrados por el MEP no son comparables con los obtenidos por el HF, esto no parece influenciar el comportamiento adhesivo del sustrato cerámico ${ }^{(7,10)}$. Por el contrario, un tiempo de aplicación más prolongado del MEP mostró resultados adhesivos superiores a la técnica convencional con HF y silano( ${ }^{(13)}$. Esto al parecer se explica por la presencia de sales de fluoruro sobre la superficie de la cerámica tras la aplicación del MEP, así como la consolidación de una monocapa altamente condensada de silano ${ }^{(9)}$, lo que favorece la unión química y reduce la degradación hidrolítica de la unión siloxana, mejorando la longevidad de la adhesión ${ }^{(10,11)}$. Como se puede observar en el control al año y medio (Figura 7), no existe pérdida de integridad ni tinción en los márgenes de la restauración, lo que se condice con los resultados in vitro a largo plazo para este materia|(11,12). Más aún, la unión adhesiva ha sobrevivido exitosamente el tratamiento de ortodoncia al que se está sometiendo actualmente la paciente, soportando sin problemas las fuerzas de tracción transmitidas por los brackets a la cerámica.

Pese a que la evidencia sobre el rendimiento clínico del MEP es limitada, los reportes de Cedillo y cols. ${ }^{(14)}$ y Siqueira y cols. ${ }^{(15)}$ considerando períodos de seguimiento de hasta 6 meses, concuerdan con lo observado en el presente caso. Estudios clínicos a mediano y largo plazo permitirán obtener una visión completa del rendimiento de estos nuevos sistemas adhesivos.

\section{CONCLUSIÓN}

La introducción de un sistema de acondicionamiento autograbante como alternativa al HF permite no sólo simplificar el proceso de cementación, sino que hacerlo más seguro y reproducible. Pese a su seguimiento relativamente corto, la evidencia aportada por este caso clínico permite considerarlo como una alternativa válida en el acondicionamiento de este tipo de sustrato.

\section{RELEVANCIA CLÍNICA}

En el presente reporte de caso se describe un innovador protocolo para la cementación de carillas ultradelgadas de disilicato de litio que permite, mediante la utilización de un agente autograbante en base a polifluoruro de amonio, evitar el uso de ácido fluorhídrico para el acondicionamiento del sustrato cerámico. De esta manera se previenen los riesgos asociados al ácido fluorhídrico, al tiempo que se facilita la técnica de cementación sin sacrificar la calidad de la adhesión lograda al disilicato de litio. Todo lo anterior se ve ejemplificado con el adecuado resultado estético del caso y la ausencia de complicaciones en los 18 meses de seguimiento del mismo.

\section{CONFLICTO DE INTERESES}

Los autores del presente trabajo no declaran ningún conflicto de interés. El trabajo clínico realizado no contó con ningún tipo de financiamiento. El tratamiento fue cancelado íntegramente por la paciente.

\section{Bibliografía}

1. Grandon F, Marcus N, Muster M. Esthetic rehabilitation with ultra-thin ceramic veneers and direct mock-up in the treatment of dental erosion - case report. J Oral Res. 2018;6: 254-259.

2. Clavijo V, Sartori N, Phark JH, Duarte S. Novel Guidelines for bonded ceramic veneers: part 1. Is tooth preparation truly necessary? En: Duarte Jr S, editor. Quintessence of Dental Technology 1st Ed. Hannover: Quintessence; 2016. 3. Yan J, Kaizer MR, Zhang Y. Load-bearing capacity of lithium disilicate and ultratranslucent zirconias. J Mech Behav Biomed Mater. 2018;88: 170-175.

4. Morimoto S, Albanesi RB, Sesma N, Agra CM, Braga MM. Main Clinical outcomes of feldspathic porcelain and glass-ceramic laminate veneers: A systematic review and meta-analysis of survival and complication rates. Int J Prosthodont. 2016;29: 38-49.

5. Ozcan M, Allahbeickaraghi A, Dundar M. Possible hazardous effects of hydrofluoric acid and recommendations for treatment approach: a review. Clin Oral Investig. 2012;16: 15-23.

6. Klosa K, Boesch I, Kern M. Long-term bond of glass ceramic and resin cement: evaluation of titanium tetrafluoride as an alternative etching agent for lithium disilicate ceramics. J Adhes Dent. 2013;15: 377-383.

7. Wille S, Lehmann F, Kern M. Durability of resin bonding to lithium disilicate and zirconia ceramic using a self-etching primer. J Adhes Dent. 2017;19: 491-496. 8. Voelkel T, Braziulis E. Monobond Etch and Prime. Scientific Documentation. Ivoclar Vivadent. 2015.

9. El-Damanhoury HM, Gaintantzopoulou MD. Self-etching ceramic primer versus hydrofluoric acid etching: Etching efficacy and bonding performance. J Prosthodont Res. 2018;62: 75-83.
10. Maier E, Bordihn V, Belli R, Taschner M, Petschelt A, Lohbauer U, et al. New approaches in bonding to glass-ceramic: self-etch glass-ceramic primer and universal adhesives. J Adhes Dent. 2019;21: 209-217.

11. Prado M, Prochnow $C$, Marchionatti AME, Baldissara $P$, Valandro LF, Wandscher VF. Ceramic surface treatment with a single-component primer: resin adhesion to glass ceramics. J Adhes Dent. 2018;20: 99-105.

12. Lyann SK, Takagaki T, Nikaido T, Uo M, Ikeda M, Sadr A, et al. Effect of different surface treatments on the tensile bond strength to lithium disilicate glass ceramics. J Adhes Dent. 2018;20: 261-268.

13. Cardenas AFM, Quintero-Calderon AS, Siqueira FSF, Campos VS, Wendlinger $\mathrm{M}$, Pulido-Mora CA, et al. Do different application modes improve the bonding performance of self-etching ceramic primer to lithium disilicate and feldspathic ceramics? J Adhes Dent. 2019;21: 319-327.

14. Cedillo Valencia J, Solano González A. Monobond Etch \& Prime, primer acondicionador monocomponente, que permite el grabado y la silanización de las superficies cerámicas en un solo paso. Rev ADM. 2018;75: 358-369.

15. Siqueira FS, Alessi RS, Cardenas AF, Kose C, Souza Pinto SC, Bandeca $\mathrm{MC}$, et al. New single-bottle ceramic primer: 6-month case report and laboratory performance. J Contemp Dent Pract. 2016;17: 1033-1039. 